Preliminary work indicates that it is possible to assess the local mechanical properties of a stent by atomic force microscopy and nano-indentation through evaluation of the unloading curves (figures 3 and 4). Further work would incorporate assessing the performance of the polymer scaffolds at different degradation time points to ascertain that vessel patency is achieved before complete degradation of BRSs.

Results obtained here will help gain a better understanding of local and global mechanical properties of BRSs and enable further research and development of the scaffolds.

Acknowledgement This work was supported by a grant from the British Heart Foundation (BHF).

\section{THE EFFECTS OF TBQ ON CARDIAC INTRACELLULAR ATP LEVELS; ROLE OF OXIDATIVE PHOSPHORYLATION AND OXIDATIVE STRESS}

${ }^{1}$ Natasha Hadgraft" ${ }^{*}$ David Greensmith, ${ }^{2}$ Gina Galli, ${ }^{3}$ Louise Miller. ${ }^{1}$ University of Salford: ${ }^{2}$ Division of Cardiovascular Science, s School of Medical Sciences, Faculty of Biology, Medicine and Health, The University of Manchester; ${ }^{3}$ Division of Cardiovascular Sciences, School of Medical Sciences, Faculty of Biology, Medicine and Health, The University of Mancheste

\subsection{6/heartjnl-2017-311726.173}

In a recent study, 2,5-Di-(tert-butyl)-1,4-benzohydroquinone (TBQ) produced a concentration dependent and fully reversible inhibition of the sarcoplasmic reticulum $\mathrm{Ca}^{2+}$ ATPase (SERCA) in rat ventricular myocytes ${ }^{1}$. While TBQ is a potentially useful research tool to study SERCA inhibition in cardiac cells, many additional effects were observed including production of an outward current consistent with activation of an ATP dependent potassium channel. The current study aims to determine the mechanisms underlying these effects.

Rat and sheep ventricular myocytes were isolated by enzymatic digestion. Intracellular ATP levels were measured using a ViaLight Plus Cell Proliferation Kit (Lonza). Mitochondrial oxygen consumption and levels of hydrogen peroxide were measured using an Oxygraph-2k high resolution respirometer (Oroboros Instruments).

In rat and sheep ventricular myocytes, TBQ produced a concentration dependent decrease of intracellular ATP where $100 \hat{\mathrm{I}}^{1} / 4 \mathrm{M}$ TBQ decreased ATP levels to approximately $60 \%$ of control. Removal of glucose from the experimental solutions had no effect on the magnitude of effect. In rat homogenates, TBQ produced a concentration dependent decrease of

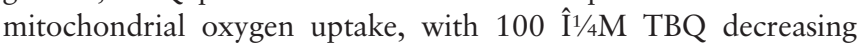
rate to $85 \%$ of control. TBQ increased levels of hydrogen peroxide, however catalase, did not attenuate TBQs effect on mitochondrial oxygen uptake.

The current findings suggest TBQ decreases intracellular ATP, a phenomenon which may account for many of the effects observed previously, including activation of an ATP dependent potassium channel. The reduction in ATP appears to be associated with an effect on oxidative phosphorylation rather than glycolysis. While TBQ is associated with an increase in hydrogen peroxide, which may increase oxidative stress, the experiments carried out with catalase suggest that this does not contribute to TBQs effect on mitochondrial function.

\section{REFERENCE}

1. Miller L, Greensmith DJ, Sankaranarayanan R, O'Neill SC, Eisner DA. The effect of 2,5-di-(tert-butyl)-1,4-benzohydroquinone (tbq) on intracellular $\mathrm{ca}^{2+}$ handling in rat ventricular myocytes. Cell calcium. 2015;58:208-214

\section{SEROTONIN RECEPTOR 2B (5-HT2B) MODULATES CARDIOMYOCYTE PROLIFERATION BY REGULATING THE HIPPO PATHWAY}

Dowan Kwon*, Yulia Kohar, Nicholas Stafford, Delvac Oceandy. Division of Cardiovascular Sciences, University of Manchester

10.1136/heartjnl-2017-311726.174

Heart failure is one of the leading causes of death worldwide. In part, this is due to the inadequate regenerative capacity of cardiomyocytes post-injury. Modulation of the Hippo signalling pathway in mice has been shown to enhance cardiomyocyte proliferation and improve survival in a myocardial infarction model. While the discovery of the Hippo pathway and its function as a master regulator of cell proliferation has led to greater understanding of its core components such as Yes-associated protein (YAP), the upstream signals that regulate the Hippo pathway have remained elusive. This study was aimed to identify novel upstream regulators of the Hippo pathway in cardiomyocytes that could be targeted pharmacologically to induce regeneration.

We performed a targeted RNAi screen in H9c2 cardiomyoblast cell line using adenovirus-mediated luciferase reporter system to detect the activity of YAP, the major effector of the Hippo pathway. Using this system, 5-hydroxytryptamine receptor $2 \mathrm{~B}\left(5-\mathrm{HT}_{2 \mathrm{~B}}\right)$ was identified as a potential regulator of the Hippo pathway. Serotonin-mediated $5-\mathrm{HT}_{2 \mathrm{~B}}$ has previously been shown to play a significant role in cardiac development during embryogenesis; however, a link between $5-\mathrm{HT}_{2 \mathrm{~B}}$ and the Hippo pathway has not yet been documented.

An in vitro model was subsequently established by overexpressing $5-\mathrm{HT}_{2 \mathrm{~B}}$ in primary neonatal rat cardiomyocytes (NRCMs) using an adenoviral system. The activities of different components of the Hippo pathway were investigated with an emphasis on YAP. Immunofluorescence microscopy was utilised to quantify cardiomyocyte proliferation and survival.

Using this system, we found that overexpression of $5-\mathrm{HT}_{2 \mathrm{~B}}$ in cardiomyocytes enhanced YAP activity by 12 folds compared to the control group as indicated by YAP-luciferase assay. In keeping with this, we observed an increase in YAP nuclear translocation following $5-\mathrm{HT}_{2 \mathrm{~B}}$ overexpression, indicating YAP activation. Mechanistically, we found that $5-\mathrm{HT}_{2 \mathrm{~B}}$ expression reduced Large tumour suppressor (LATS) phosphorylation, eventually leading to YAP activation. Since YAP is known to mediate cell proliferation we analysed proliferation rate in cardiomyocytes overexpressing $5-\mathrm{HT}_{2 \mathrm{~B}}$. We found that cell proliferation was increased by $39.7 \%$ compared with control cells as indicated by EdU incorporation assay.

In conclusion, our findings have identified $5-\mathrm{HT}_{2 \mathrm{~B}}$ as a novel upstream regulator of the Hippo pathway in cardiomyocytes. We also observed that in cardiomyocytes, $5-\mathrm{HT}_{2 \mathrm{~B}}$ is a potent stimulator of YAP activity and cell proliferation. Since $5-\mathrm{HT}_{2 \mathrm{~B}}$ is a membrane receptor that can be targeted pharmacologically, this finding may provide new insight for the development of a new approach to induce cardiomyocyte regeneration. 